Pacific

Journal of

Mathematics

ON THE ADDITIVITY OF THE THURSTON-BENNEQUIN INVARIANT OF LEGENDRIAN KNOTS

ICHIRO TORISU 


\title{
ON THE ADDITIVITY OF THE THURSTON-BENNEQUIN INVARIANT OF LEGENDRIAN KNOTS
}

\author{
ICHIRO TORISU
}

\begin{abstract}
In this article, we consider the maximal value of the Thurston-Bennequin invariant of Legendrian knots which topologically represent a fixed knot type in the standard contact 3-space and we prove a formula of the value under the connected sum operation of knots.
\end{abstract}

\section{Introduction.}

The standard contact structure $\xi_{0}$ on 3 -space $\mathbb{R}^{3}=\{(x, y, z)\}$ is the plane field on $\mathbb{R}^{3}$ given by the kernel of the 1 -form $d z-y d x$. A Legendrian knot $K$ in the contact manifold $\left(\mathbb{R}^{3}, \xi_{0}\right)$ is a knot which is everywhere tangent to the contact structure $\xi_{0}$. The Thurston-Bennequin invariant $\operatorname{tb}(K)$ of a Legendrian knot $K$ in $\left(\mathbb{R}^{3}, \xi_{0}\right)$ is the linking number of $K$ and a knot $K^{\prime}$ which is obtained by moving $K$ slightly along the vector field $\frac{\partial}{\partial z}$. For a topological knot type $k$ in $\mathbb{R}^{3}$, the maximal Thurston-Bennequin invariant $\operatorname{mtb}(k)$ is defined to be the maximal value of $\operatorname{tb}(K)$, where $K$ is a Legendrian knot which topologically represents $k$. For any $k$, by the Bennequin's inequality in $[\mathbf{1}]$, we know that $\operatorname{mtb}(k)$ is an integer (i.e., not $\infty$ ). There are several computations of $\operatorname{mtb}(k)$ (for example, see [3], [5], [8], [9], [10], [11]).

In this paper, we prove the following theorem:

Theorem 1.1. Let $k_{1} \sharp k_{2}$ be the connected sum of topological knots $k_{1}$ and $k_{2}$ in $\mathbb{R}^{3}$. Then $\operatorname{mtb}\left(k_{1} \sharp k_{2}\right)=\operatorname{mtb}\left(k_{1}\right)+\operatorname{mtb}\left(k_{2}\right)+1$.

Remark 1.2. After writing this paper, the author was informed that J. Etnyre and K. Honda [4] have also obtained a result on connected sum of Legendrian knots which extensively includes Theorem 1.1 and that T. Tanaka [12] have partially proved Theorem 1.1 by using a technique of algebraic knot theory.

\section{Fronts.}

Let $K$ be a Legendrian knot in $\left(\mathbb{R}^{3}, \xi_{0}=\operatorname{ker}(d z-y d x)\right)$. Then a diagram (i.e., projection) of $K$ in $x z$-plane is called front as in Figure 1.

A front does not have vertical tangents; generically, its only singularities are transverse double points and semicubical cusps. Note that the number 


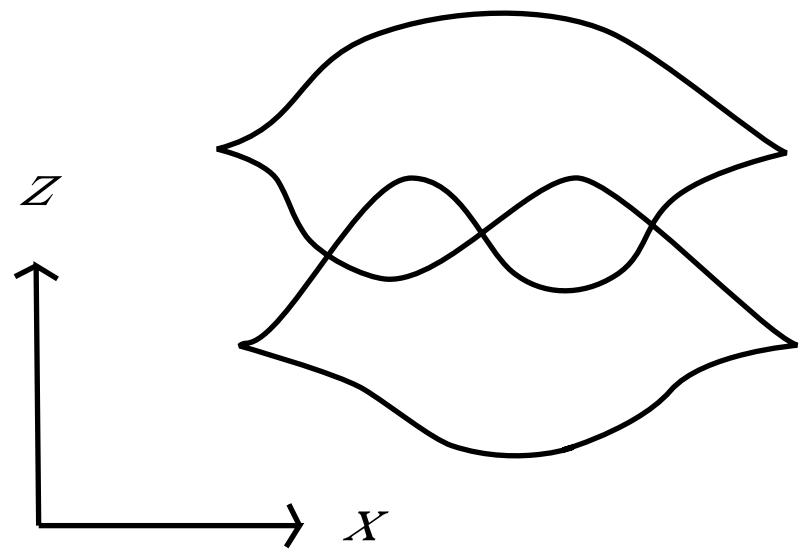

Figure 1.

of the cusps is even. Since $y=\frac{\partial z}{\partial x}$ along $K$, the missing $y$ coordinate is the slope of the front. Therefore the front of $K$ is free from selftangencies, and, at a double point, the branch with a greater slope is higher along the $y$ axis. Conversely such a diagram uniquely determines $K$ as its front. So, as usual in knot theory, we identify a Legendrian knot $K$ with its front, also denoted by $K$.

The Thurston-Bennequin invariant $\operatorname{tb}(K)$ is computed in terms of the double points and cusps of its front. See Figure 2, where $K$ is oriented and the choice of the orientaion is irrelevant for the value of $\operatorname{tb}(K)$.

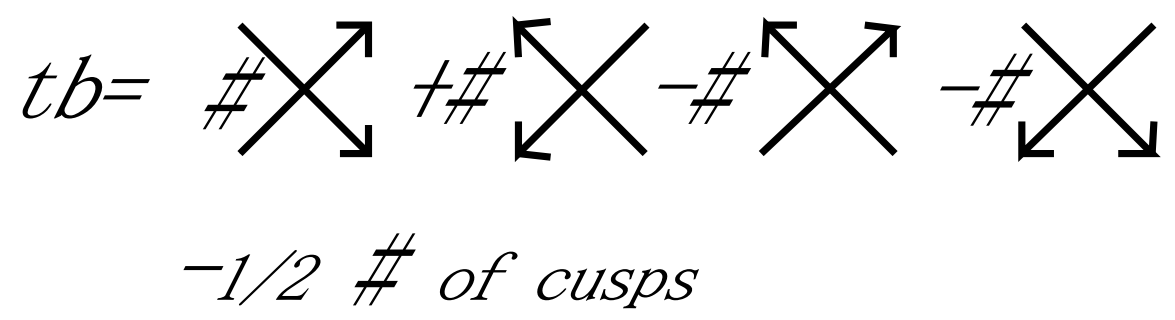

Figure 2.

For example, $\operatorname{tb}(K)=1$ for the front in Figure 1.

Proposition 2.1. For two topological knots $k_{1}$ and $k_{2}$, we have $\operatorname{mtb}\left(k_{1} \sharp k_{2}\right)$ $\geq \operatorname{mtb}\left(k_{1}\right)+\operatorname{mtb}\left(k_{2}\right)+1$.

Proof. Let $K_{1}$ and $K_{2}$ be Legendrian knots whose topological types are $k_{1}$ and $k_{2}$, respectively and $\operatorname{mtb}\left(k_{1}\right)=\operatorname{tb}\left(K_{1}\right)$ and $\operatorname{mtb}\left(k_{2}\right)=\operatorname{tb}\left(K_{2}\right)$. We also regard $K_{1}$ and $K_{2}$ as fronts. Further we can assume that $K_{1} \cap K_{2}=\emptyset$ and $K_{1}$ 

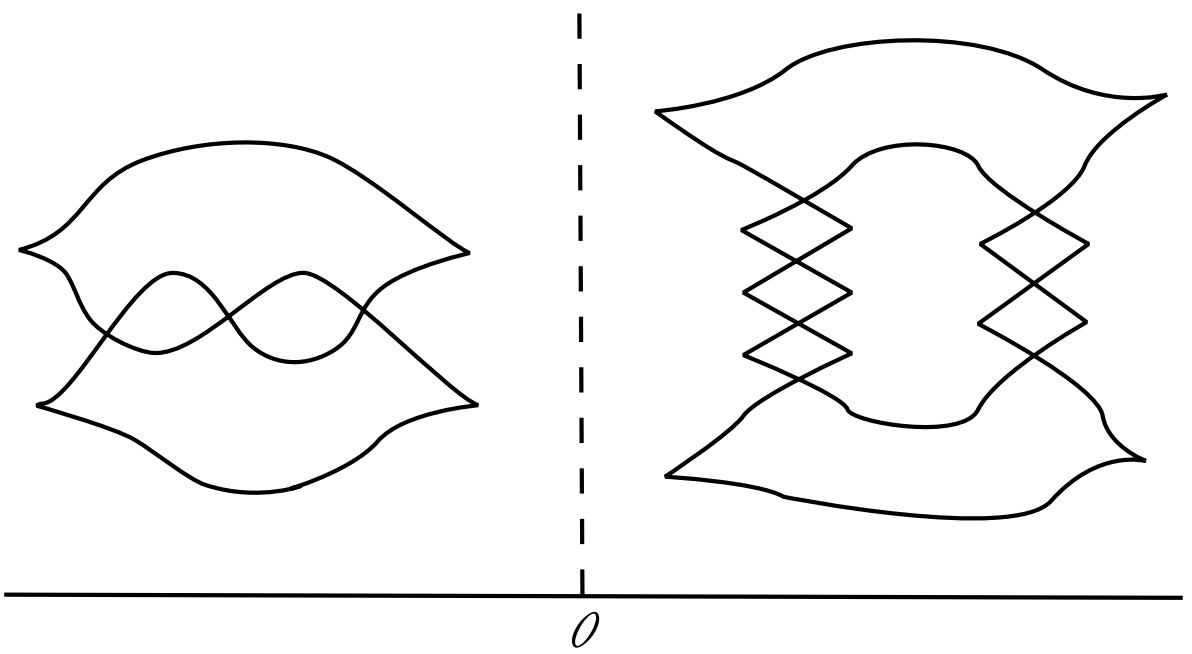

Figure 3.

(resp. $\left.K_{2}\right)$ lies in the left (resp. right) region of $x z$-plane, i.e., $\{(x, z) \mid x<0\}$ (resp. $\{(x, z) \mid x>0\})$ as in Figure 3.

Then we connect $K_{1}$ and $K_{2}$ by joining a right cusp of $K_{1}$ and a left cusp of $K_{2}$ as in Figure 4.

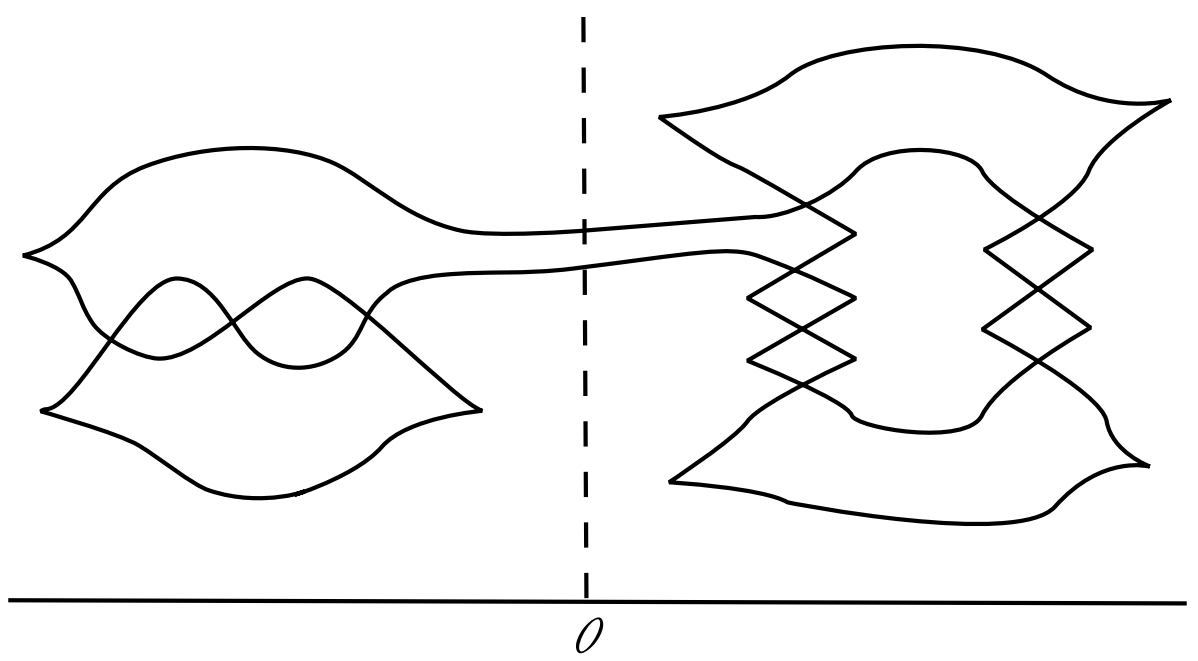

Figure 4. 
This procedure produces a Legendrian knot whose topological type is $k_{1} \sharp k_{2}$ and Thurston-Bennequin invariant is $\operatorname{mtb}\left(k_{1}\right)+\operatorname{mtb}\left(k_{2}\right)+1$. This completes the proof.

\section{Preliminaries from contact topology.}

In this section, we recall some basic notions and theorems from recent 3-dimensional contact topology. In fact, the proof of Theorem 1.1 essentially relies on the previous foundational work of E. Giroux, Honda and Y. Eliashberg-M. Fraser. In particular, we assume the reader is familiar with convex surface theory started by Giroux in [6]. For details and proofs, see $[\mathbf{2}],[\mathbf{3}],[\mathbf{6}],[\mathbf{7}],[\mathbf{8}]$. Let $\xi_{n}=\operatorname{ker}(\sin (2 \pi n z) d x+\cos (2 \pi n z) d y)$ be the contact structure on a solid torus $V=\left\{(x, y, z) \in \mathbb{R}_{z}^{3} \mid x^{2}+y^{2} \leq \epsilon\right\}$, where $n \in \mathbb{N}$ and $\mathbb{R}_{z}^{3}$ is $\mathbb{R}^{3}$ modulo $z \mapsto z+1$. The characteristic foliation on an embedded surface in a contact 3-manifold is the singular foliation defined by the intersection of the contact structure and the surface. The set of tangents of $\xi_{n}$ to $\partial V$ forms a disjoint union of two simple closed curves on $\partial V$, which are called Legendrian divides.

The next lemma is proved by a standard Darboux-type argument.

Lemma 3.1. For any Legendrian knot $K$ in $\left(\mathbb{R}^{3}, \xi_{0}\right)$, there exists a sufficiently small neighborhood $N(K)$ such that $\left(N(K), K, \xi_{0}\right)$ is isomorphic to $\left(V,\{(0,0, z)\}, \xi_{n}\right)$ for some $n$.

As $\partial V$ is a convex surface (i.e., has a contact vector field transverse to $\partial V)$, the following lemma can be proved by convex surface theory:

Lemma 3.2. Let $T$ be any embedded torus in $\left(\mathbb{R}^{3}, \xi_{0}\right)$ and $W$ a solid torus bounded by $T$. Suppose the characteristic foliation on $T$ is diffeomorphic to that on $\partial V$ and identifying these, the Legendrian divides on $T$ are isotopic to the core curve of $W$ through an isotopy in $W$. Then $\left(W, \xi_{0}\right)$ is isomorphic to $\left(V, \xi_{n}\right)$ for some $n$.

The following theorem on the classification of topologically trivial Legendrian knots due to Eliashberg-Fraser [2] is also needed for the proof of Theorem 1.1:

Theorem 3.3. Any topologically trivial Legendrian knot is Legendrian isotopic to one of standard forms expressed as fronts in Figure 5.

\section{Proof of Theorem 1.1.}

By Proposition 2.1, it is sufficient to show the converse inequality.

Suppose $\hat{K}$ is a Legendrian knot in $\left(\mathbb{R}^{3}, \xi_{0}\right)$ whose topological type is the connected sum of $k_{1}$ and $k_{2}$ and its Thurston-Bennequin invariant is maximal. By Lemma 3.1, there exists a neighbourhood $N(\hat{K})$ of $\hat{K}$ such 


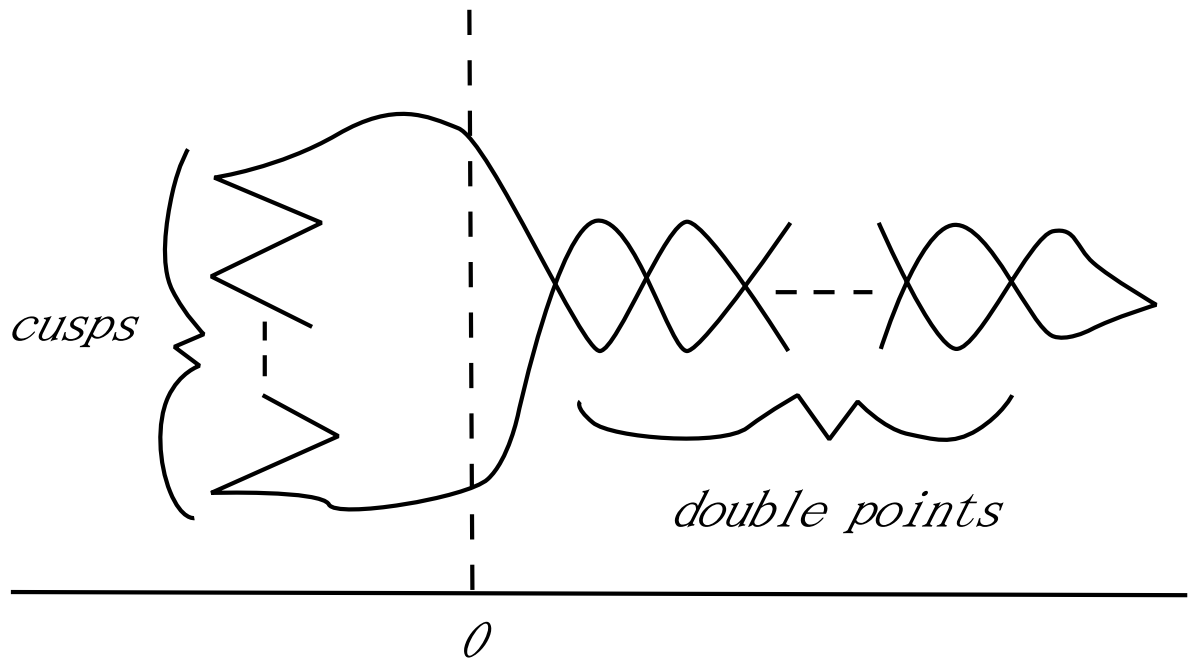

Figure 5 .

that $\left(N(\hat{K}), \xi_{0}\right)$ is isomorphic to $\left(V, \xi_{n}\right)$ for some $n$. Let $B_{1}$ and $B_{2}$ be 3-balls in $\mathbb{R}^{3}$ such that $B_{1}$ (resp. $B_{2}$ ) splits $\hat{K}$ into the component corresponding to $k_{1}$ (resp. $k_{2}$ ) and $B_{1} \cap B_{2}=\emptyset$ (Figure 6).

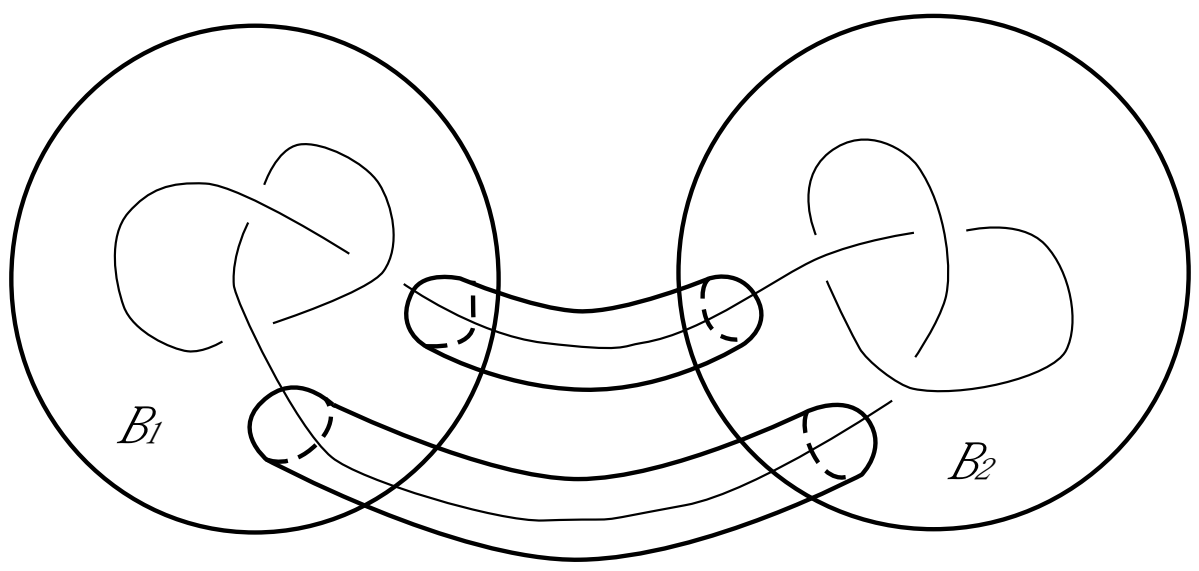

Figure 6.

Further, by convex surface theory, we can assume that (i) $\partial B_{1}$ and $\partial B_{2}$ are convex and (ii) $\partial B_{1} \cap \partial N(\hat{K})$ and $\partial B_{2} \cap \partial N(\hat{K})$ are Legendrian knots on $\partial B_{1}$ and $\partial B_{2}$, respectively and (iii) each dividing set on $\partial B_{i}$ (i.e., the subset of $\partial B_{i}$ consisting of tangents of $\xi_{0}$ and a contact vector field defining the convex surface) intersects $\partial B_{i} \cap N(\hat{K})$ as a diameter of the disk. 
Then by Edge-Rounding Lemma due to Honda in [7], we have a solid torus $W$ such that (i) $W$ equals $B_{1} \cup B_{2} \cup N(\hat{K})$ except small neighbourhoods of $\partial B_{1} \cap \partial N(\hat{K})$ and $\partial B_{2} \cap \partial N(\hat{K})$ and (ii) $\partial W$ is a convex surface whose characteristic foliation is diffeomorphic to that of $\partial V$. By Lemma 3.2, it follows that $\left(W, \xi_{0}\right)$ is isomorphic to $\left(V, \xi_{n}\right)$ for some $n$. And notice that $W$ is unknotted in $\mathbb{R}^{3}$ and hence the core curve $K$ of $W$ which is Legendrian is also unknotted. Further, by a standard argument, we can assume that $K$ agrees with $\hat{K}$ in the region of $N(\hat{K})-\left(B_{1} \cup B_{2}\right)$. So by Theorem 3.3, $K$ is Legendrian isotopic to one of standard forms in Figure 5. Therefore $W$ is also identified with a small neighbourhood of that of the standard form. Further, by a homogeneous property of $V$ and a parallel translation of $W$, we can assume that a region of $W$ corresponding to $B_{1}$ (resp. $B_{2}$ ) lies in $\{(x, y, z) \mid x<0\}$ (resp. $\{(x, y, z) \mid x>0\}$ ). Then, identifying $\hat{K}$ with its front, we can divide $\hat{K}$ along a vertical line into Legendrian knots $K_{1}$ and $K_{2}$ corresponding to $k_{1}$ and $k_{2}$, respectively as the converse procedure in the proof of Proposition 2.1.

Counting the Thurston-Bennequin invariant of $K_{1}$ and $K_{2}$, we have $\operatorname{tb}(\hat{K})$ $=\operatorname{mtb}\left(k_{1} \sharp k_{2}\right)=\operatorname{tb}\left(K_{1}\right)+\operatorname{tb}\left(K_{2}\right)+1$. Therefore $\operatorname{mtb}\left(k_{1} \sharp k_{2}\right) \leq \operatorname{mtb}\left(k_{1}\right)+$ $\operatorname{mtb}\left(k_{2}\right)+1$.

This completes the proof of the main theorem.

\section{References}

[1] D. Bennequin, Entrelacements et équations de Pfaff, Astérisque, 107-108 (1983), 87-161, MR 86e:58070, Zbl 0573.58022.

[2] Y. Eliashberg and M. Fraser, Classification of topologically trivial Legendrian knots, in 'Geometry, Topology and Dynamics' (Montreal, PQ, 1995), CRM Proc. Lecture Notes, 15 (1998), 17-51, MR 99f:57007, Zbl 0907.53021.

[3] J. Etnyre and K. Honda, Knots and contact geometry I: Torus knots and the figure eight knot, preprint.

[4] K Knots and contact geometry II: Connected sums, preprint.

[5] D. Fuchs and S. Tabachnikov, Invariants of Legendrian and transverse knots in the standard contact space, Topology, 36 (1997), 1025-1053, MR 99a:57006, Zbl 0904.57006.

[6] E. Giroux, Convexité en topologie de contact, Comment. Math. Helvetici, 66 (1991), 637-677, MR 93b:57029, Zbl 0766.53028.

[7] K. Honda, On the classification of tight contact structures I, Geom. Topol., 4 (2000), 309-368, MR 2001i:53148, Zbl 0980.57010.

[8] Y. Kanda, On the Thurston-Bennequin invariant of Legendrian knots and non exactness of Bennequin's inequality, Invent. Math., 133 (1998), 227-242, MR 99g:57030, Zbl 0902.57007.

[9] L. Rudolph, An obstruction to sliceness via contact geometry and "classical" gauge theory, Invent. Math., 119 (1995), 155-163, MR 95k:57013, Zbl 0843.57011. 
[10] S. Tabachnikov, Estimates for the Bennequin number of Legendrian links from state models for knot polynomials, Math. Research Letters, 4 (1997), 143-156, MR 98k:57023, Zbl 0877.57001.

[11] T. Tanaka, Maximal Bennequin numbers and Kauffman polynomials of positive links, Proc. Amer. Math. Soc., 127 (1999), 3427-3432, MR 2000b:57014, Zbl 0983.57008.

[12] _ On invariants of Legendrian links, preprint.

Received July 12, 2001 and revised June 27, 2002.

Department of Computer Science And Engineering

AKITA University

1-1 Tegata GaKuen-ChO

AKITA 010-8502

JAPAN

E-mail address: torisu@math.akita-u.ac.jp 